\title{
Theoretical study on effect of radial and axial deformation on electron transport properties in a semiconducting $\mathrm{Si}-\mathrm{C}$ nanotube
}

\author{
SUDHANSHU CHOUDHARY* and S QURESHI \\ Department of Electrical Engineering, Indian Institute of Technology, Kanpur 208 016, India
}

MS received 26 April 2011; revised 13 February 2012

\begin{abstract}
We investigate electron transport properties in a deformed $(8,0)$ silicon carbide nanotube by applying self consistent non-equilibrium Green's function formalism in combination with the density-functional theory to a two-probe molecular junction constructed from deformed nanotube. The results suggest significant reduction in threshold voltage in the case of both radially compressed and axially elongated $(8,0)$ SiCNTs, a large difference in current-voltage characteristics was observed. Analysis of frontier molecular orbitals (FMO) and transmission spectrum show bandgap reduction in deformed nanotubes. Deformation introduces electronic states near the Fermi level, enhancing the conduction properties of $(8,0)$ SiCNT. The FMOs and the orbitals corresponding to peaks in $T(E)$ around Fermi level obviously has some major contributions from the deformed site. However, localization of the electronic state near the Fermi level is weak in $(8,0)$ SiCNT, possibly because of its large bandgap.
\end{abstract}

Keywords. Nanotube; SiCNT; armchair-zigzag; defects; deformation.

\section{Introduction}

Silicon carbide nanotubes have a great potential for application in chemical and biological sensors (Taguchia et al 2005a; Xie et al 2007). Recently, scientists have suggested to make silicon carbide nanotubes (SiCNTs) as a possible alternative to carbon nanotubes (CNTs) for field emitting applications (Taguchia et al 2005b; Alfieri and Kimoto 2010), which has boosted the interest for SiCNTs and several studies on the electronic properties of SiCNTs can be found in the literature (Mavrandonakis et al 2003; Mpourmpakis et al 2006; Larina et al 2007; Muralidharan et al 2007; Wu and Guo 2007; Lakshmi et al 2008; Moradian et al 2008; Raidongia et al 2008; Voggu et al 2008; Wu et al 2008; Manna and Pati 2010; Zheng et al 2010). It is well known that CNTs undergo a semiconductor to metal transition (SMT) (Ren et al 2009) or metal to semiconductor transition (MST) (Lu et al 2003) when deformed. Therefore, it is of interest to understand how the electronic properties of SiCNTs would get altered when deformed.

It is known that SiCNTs are generally semiconductors, except for the $(3,0)$ and $(4,0)$ nanotubes which are metals (Wu and Guo 2007), and the bandgap $\left(E_{\mathrm{g}}\right)$ is wider for larger tube diameters (Zhao et al 2005; Alfieri and Kimoto 2010). However, another study (Larina et al 2007) suggests that in the range $n=7-10,(n, n)$ nanotubes are semiconductors and their bandgap decreases steadily with increasing $n$, nanotubes with $n=5$ and 6 are metallic. Also, at $n=7-9,(n, 0)$

\footnotetext{
*Author for correspondence (suds@iitk.ac.in)
}

nanotubes are semiconductors, and their bandgap increases steadily with $n$, nanotubes with $n=5$ and 6 have metallic conductivity. An applied uniaxial strain results in the modification of both $E_{\mathrm{g}}$ width and band structure of SiCNTs (Wang et al 2008) which was also observed for CNTs (Alfieri and Kimoto 2010), the effects of radial compression along with axial elongation on the electronic properties of SiCNTs were reported recently in our previous research work (Choudhary and Qureshi 2011).

The traditional methods find difficulty in studying the transport properties of SiCNTs because of their small feature size. However, the method combined with density functional theory (DFT) and non-equilibrium Green's function (NEGF) have been applied successfully to investigate the electron transport and other properties (Song et al 2009; Yang et al 2010). In the present work, we study the frontier molecular orbitals (FMO), density of states (DOS) and transmission energy spectrum, $T(E)$, to find the transport properties of $(4,0)$ and $(5,5)$ SiCNTs under both radial compression and axial elongation. Bias voltage dependent current $(I-V)$ characteristics are also studied in relation with FMOs, DOS and $T(E)$.

\section{Materials and methods}

A perfect (non-deformed) SiCNT is shown in figure 1(a) with Si-C bond length of $1.78 \AA$ (Lu et al 2003; Alfieri and Kimoto 2010) and 1:1 $\mathrm{Si}-\mathrm{C}$ ratio, whereas in figure $1(\mathrm{~b})$, a radially deformed nanotube compressed in $X Y$-coordinates ( $X Y$-distortion) is shown. The degree of radial compression or $X Y$-distortion is determined by $X Y$ ratio of the ellipse. When the tube is stretched in $Z$-coordinate ( $Z$-distortion), 


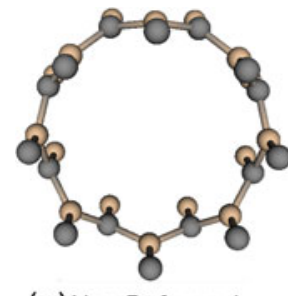

(a) Non Deformed

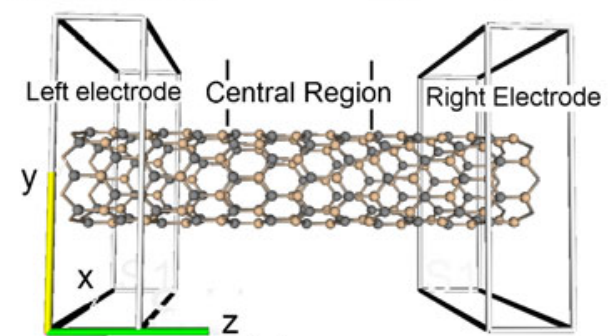

(c) Two probe junction

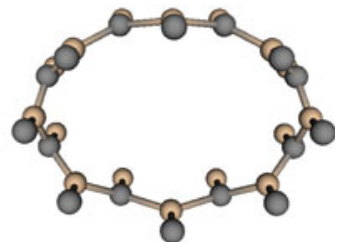

(b) Radially deformed
Figure 1. $(8,0)$ SiCNT (a) non-deformed, (b) radially deformed and (c) two-probe system of deformed $(8,0) \mathrm{SiCNT}$ with a central region and left-right electrodes.

an axially elongated or axially deformed nanotube results. The stretch factor in $Z$-coordinate determines the amount of elongation deformation. We used nanotube Modeller (www.jcrystal.com) for creating the above kind of deformed SiCNT structures, which generates $X Y Z$-coordinates of nanotubes. The above structures were exported to Atomistix (www.quantumwise.com) for setting up a two-probe geometry which consists of a left electrode, right electrode and a central region (figure 1(c)).

The simulation parameters were selected to provide accurate measurements as reported for CNTs (Yamacli and Avci 2009) and are the following: mesh cut-off energy was $400 \mathrm{Ry}$, basis set was double zeta polarized with 0.001 Bohr radial sampling, exchange correlation functional was set to local density approximation (LDA) type with double zeta polarized (DZP) basis set, Brillouin zone integration parameters of electrodes are taken as $(1,1,500)$. Electrode temperature was set to $1000 \mathrm{~K}$ which makes the convergence easier; it has no effect on the overall measurement which was also verified at lower electrode temperatures. Due to the requirement of heavy computing resources, we used the above parameters which shall ensure convergence.

To obtain transmission spectrum and $I-V$ characteristics, self consistent calculation are performed on an $a b$ initio based simulator called Atomistix which uses density functional theory (DFT) and non-equilibrium Green's functions formulations (NEGF) together for obtaining electronic transport properties of molecules and devices. More details about the method and software could be found in previous reports (Taylor et al 2001; Brandbyge et al 2002; Soler et al 2002). The transmission spectrum describes the probability for electron with incident energy $(E)$ to transfer from the left electrode to the right electrode under applied bias voltage $(V)$. The analytical expression for calculating the transmission spectrum is given by (Gali 2006; Li and Kosov 2006; Yang et al 2010)

$$
T(E, V)=\operatorname{Tr}\left[\Gamma_{\mathrm{L}}(E, V) G^{\dagger}(E, V) \Gamma_{\mathrm{R}}(E, V) G(E, V)\right],
$$

where $\Gamma_{\mathrm{L} / \mathrm{R}}$ is the coupling matrix.

The integral of transmission spectrum yields the current through the system (Gali 2006; Lakshmi et al 2008; Li and Kosov 2006; Yang et al 2010), given by:

$$
I(V)=\int_{\mu_{\mathrm{L}}}^{\mu_{\mathrm{R}}} T(E, V)\left(f\left(E-\mu_{\mathrm{L}}\right)-f\left(E-\mu_{\mathrm{R}}\right)\right) \mathrm{d} E,
$$

where $\mu_{\mathrm{L}}=-V / 2\left(\mu_{\mathrm{R}}=V / 2\right)$ is the chemical potential of the left/right electrode.

The electron transport characteristic of a SiCNT may depend on its length, resistance, contacts and applied bias. We neglect length dependency by considering a minimal length segment of nanotube in the central region. In order to minimize the contact dependency, SiCNT electrodes with deformation similar to the central region are used to construct the two-probe system (figure 1(c)). Note that since we are using semiconducting $(8,0)$ SiCNT electrodes, initially there will be no current in the system. But in the case of deformed SiCNTs with deformed electrodes there will be current because the deformation changes the conducting properties of $(8,0) \mathrm{SiCNT}$ in the central region as well as $(8,0) \mathrm{SiCNT}$ electrodes, which will be shown in §3.2. Equilibrium transport properties are not expected to differ much compared to the non-equilibrium transport properties. Our assumption of semiconducting electrodes (to minimize the contact dependency) will certainly not match experimental values of the current obtained using metallic electrodes, but is fair enough to comment on the changes in the conducting properties of SiCNT under applied deformation.

The lengths of the central regions, screening layers and electrodes of the simulated sections are taken as 8 periods (32 $\mathrm{C}$ atoms and $32 \mathrm{Si}$ atoms), 2 periods (16 C atoms and $16 \mathrm{Si}$ atoms) and 2 periods $(16 \mathrm{C}$ atoms and $16 \mathrm{Si}$ atoms), respectively. Hence, a central region width of $9.79 \AA$ (SiCNT length) for non-deformed and radially deformed case, $10.28 \AA$ for $Z 1.05$ and $10.77 \AA$ for $Z 1.1$ axial elongation cases, are used in the simulation.

\section{Electron transport results and analysis}

\subsection{Equilibrium transport properties}

The transmission spectrum, $T(E)$, is plotted in figure 2 by black curve, here the Fermi energy is set as $0 \mathrm{eV}$ (Brandbyge et al 2002), DOS is shown by red curve. The equilibrium transport properties (no bias voltage applied) of SiCNTs are studied first. A perfect $(8,0)$ SiCNT is semiconducting in nature (Alfieri and Kimoto 2010), which is confirmed by the transmission gap (transmission coefficient is zero) around Fermi level for semiconducting tube in figure 2.

The $T(E)$ for non-deformed case is shown in figure 2(a) which reflects the semiconducting nature of SiCNT with a 


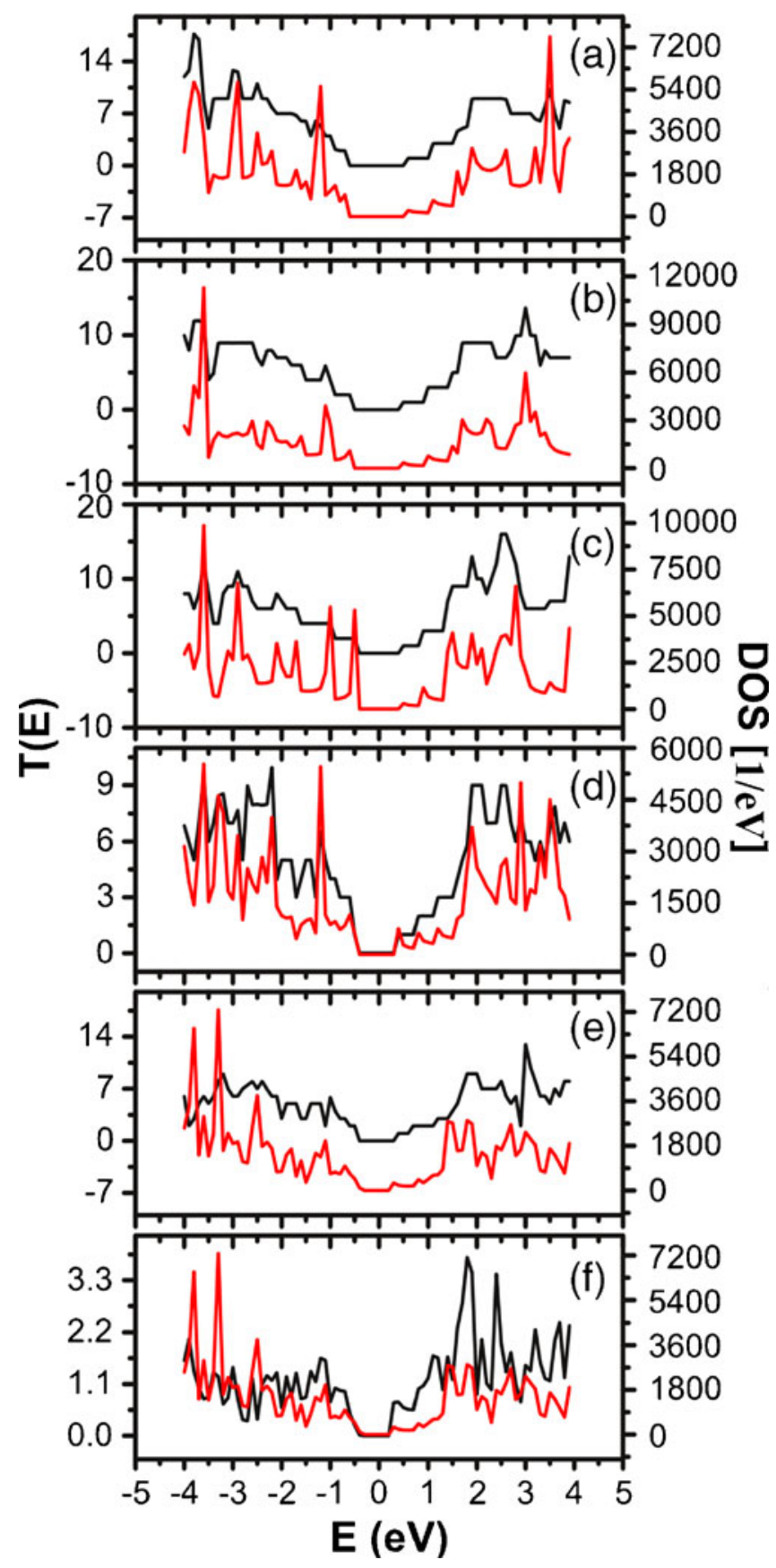

Figure 2. Zero bias transmission spectra, $T(E)$ and density of states (DOS) profile of $(8,0)$ SiCNT (a) non-deformed, (b)-(c) axial stretching deformation with stretch factor, $Z 1.05$ and $Z 1 \cdot 1$, respectively, (d) radial compression deformation with $X Y$-distortion as $X Y$ 1.3 and (e)-(f) both radial and axial deformations applied simultaneously as $X Y 1.3 Z 1.05$ and $X Y 1.5 Z 1.05$, respectively. [Note: a.u. is arbitrary units and $E$ the energy in electron volts].

wide transmission gap seen as a flat curve around Fermi level $(0 \mathrm{eV})$. Figures 2(b)-(f) presents $T(E)$ and DOS with modulation in deformation. Small reduction in transmission gap around Fermi level was observed with the increase in radial $(X Y)$ deformation, which is due to introduction of electronic states near the Fermi level with applied deformation, enhancing the conducting properties.

The bandgap variation with deformation is attributed to the change of electron orbital overlap caused by the bond length variation under deformation, which was also stated by Wang et al (2008). The radial and axial deformation destruct the $\pi$ conjugated system of the $(8,0)$ SiCNT as revealed by the frontier molecular orbitals (FMO) of the molecular projected self-consistent hamiltonian (MPSH) as shown in figures 3(b-f); here red and blue colours indicate the positive and negative wave phase in the lobe. FMOs of the $(8,0)$ SiCNT and peaks in $T(E)$ around Fermi level obviously has some major contributions from the defect site. However, localization of the electronic state is not obvious near the Fermi level, possibly because of its large bandgap. According to the analysis of figure 3 , as the deformation is increased more orbitals contribute in the current conduction (see figures 3(a) and (f)). FMOs in deformed tube are delocalized over the entire nanotube structure (see figure 3(f)) compared to strong localization near the electrodes in a perfect $(8,0) \mathrm{SiCNT}$ system. This facilitates tunneling from left to right electrode via pi-orbitals of deformed $(8,0) \mathrm{SiCNT}$, enhancing its conducting properties.

\subsection{Non-equilibrium transport properties}

We study bias voltage dependent current $(I-V)$ characteristics, which is one of the most important non-equilibrium transport characteristics (see figure 4). An axial elongation with a stretch factor of $Z 1.05$ ( $Z$-distortion) results in a small increment in current compared to non-deformed case. Nanotube was further stretched to $Z 1 \cdot 1$ to see any change in current, a small increment was observed. Next, we radially deformed SiCNT with $X Y$-distortion of $X Y 1.3$ and observed orders of magnitude of increment in current. A small increment in current was further observed when both radial and axial deformations ( $X Y 1.3 \mathrm{Z} 1.05)$ were applied simultaneously. The radial deformation was further increased to $X Y 1.5$ while axial elongation fixed at $Z 1 \cdot 05$, large increment in current was observed for both low and high bias voltages which suggest a reduced threshold in SiCNTs. However, semiconductor to metal transition (SMT) was not observed here, whereas, a SMT was demonstrated in the case of CNTs in literature (Ren et al 2009). SiCNTs could have been deformed further to observe any possibility of SMT but further deformation results in breaking of $\mathrm{Si}-\mathrm{C}$ bonds. Note that since we used electrodes similar to the central region for neglecting any contact dependency, current values obtained are lower than the experimental values obtained using metallic electrodes; choice of electrodes does not affect the objectives of the study.

For further investigation of high current characteristic, we studied the transmission spectra at a bias voltage of $2 \mathrm{~V}$ and compare it with the non-deformed case. Transmission spectra for non-deformed SiCNT and deformed case with $X Y 1.5 \mathrm{Z}$ 
(a)

(b)

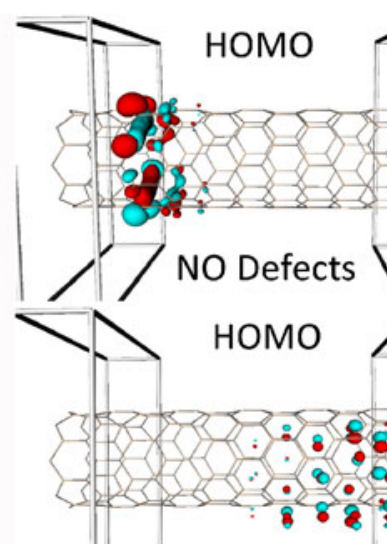

(c)

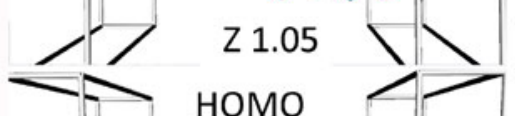

(d)

(e)

(f)

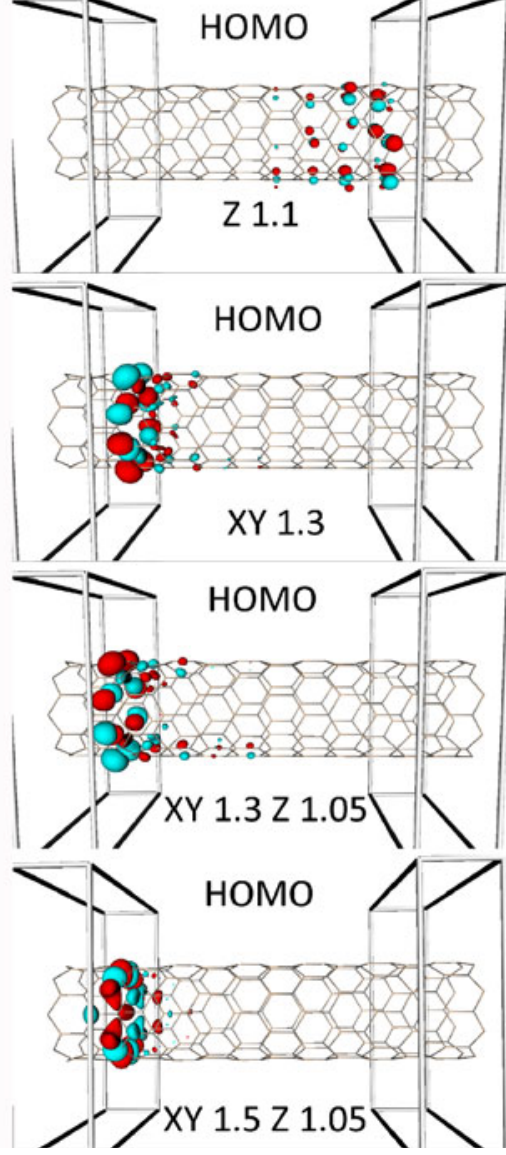

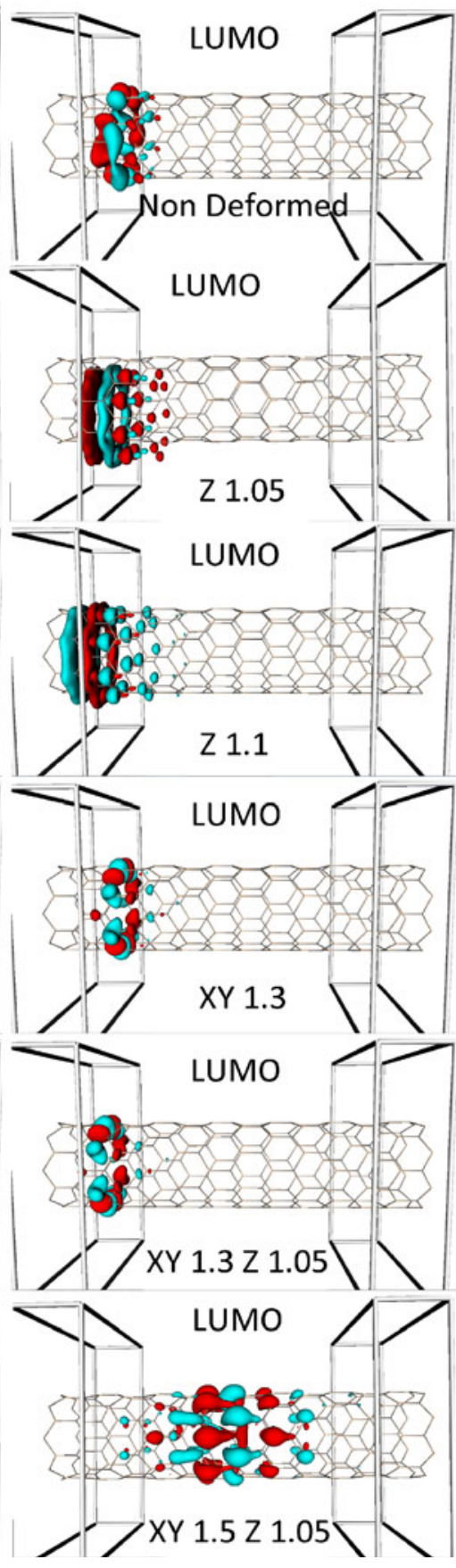

Figure 3. Frontier molecular orbitals of MPSH corresponding to $(8,0)$ SiCNT: (a) with no defects; (b-f) under applied radial and axial deformation. More FMOs contribute to conduction in deformed structures; FMOs are weakly localized that facilitates tunneling.

1.05 distortion are shown in figure 5. For non-deformed case, we see that transmission gap around the Fermi level is nearly flat, but there are peaks in case of deformed SiCNT, which indicates that new electronic states are introduced around the Fermi level. The introduction of electronic states around Fermi level suggests further reduction in bandgap, resulting in an increase in current and a reduction in threshold voltage as observed in the $I-V$ characteristic of figure 4 . These peaks in $T(E)$ contributes toward HOMO and LUMO formations.

The eigenstates of molecular projected self-consistent Hamiltonian (MPSH) are closely related to the poles of Green's function, which roughly corresponds to the peak $(p 1)$ of the transmission spectrum ( $\mathrm{Li}$ and Kosov 2006) as shown in figure 5. Frontier molecular orbitals of MPSH are 


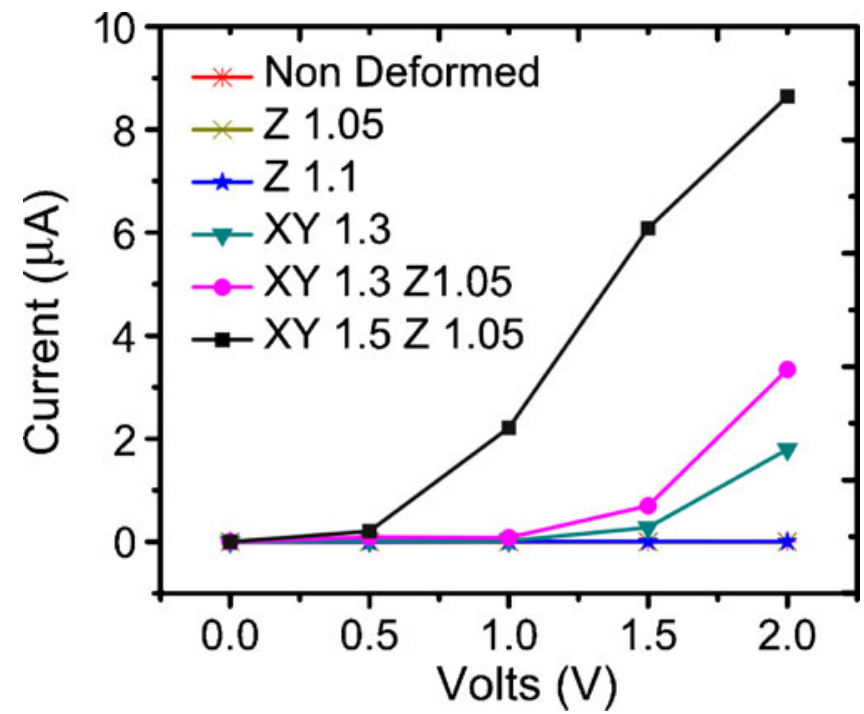

Figure 4. Bias voltage dependent current characteristic ( $I-V$ characteristic) of $(8,0) \mathrm{SiCNT}$ two-probe system. Current increases with increase in radial and axial deformation; threshold voltage reduces.

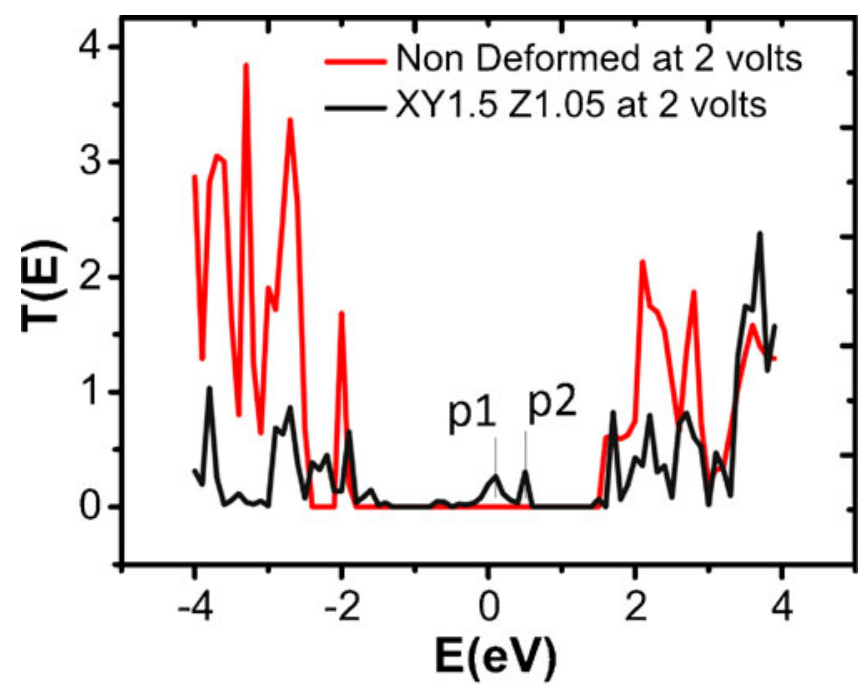

Figure 5. (a) Zero bias transmission spectra and (b) transmission spectra at $2 \mathrm{~V}$. Fermi energy is set as $0 \mathrm{eV}$. Peaks in $T(E)$ suggests introduction of new electronic states at Fermi level.

calculated and shown in figure 6 to study the physical origin of these peaks. The peak $p 1$ originates from orbital $\mathrm{O} 1$ (see figure 6) which obviously has significant contribution to the conduction properties of deformed $(8,0)$ SiCNT. An increase in current with increase in bias voltage implies that more molecular orbitals contribute to the formation of the current (Li and Kosov 2006), as shown by FMOs of MPSH in figure 6.

The results obtained from this study indicate that deformation in $(8,0)$ semiconducting SiCNT reduces the bandgap, introduces new electronic states near the Fermi level and enhances the current conduction in SiCNT. Threshold

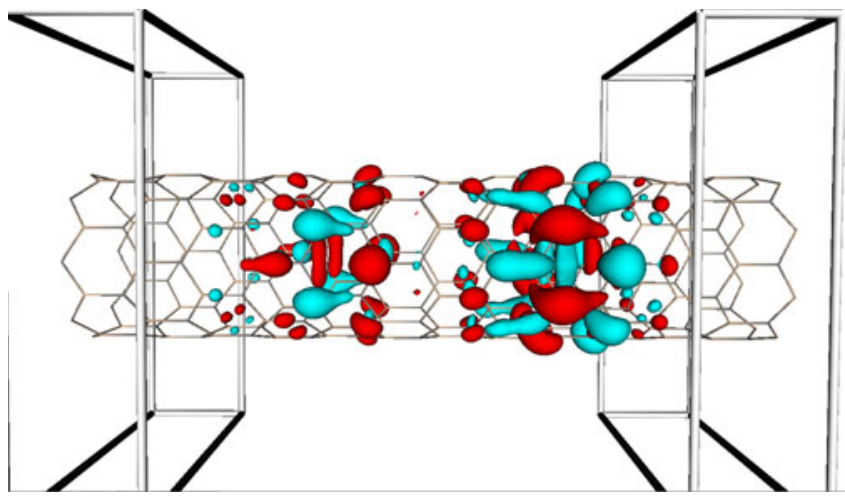

Figure 6. Orbital $(O 1)$ corresponding to peak $(p 1)$ around Fermi level in transmission spectrum of figure 4. More molecular orbitals contribute to formation of current at high bias.

voltage reduction with applied deformation could be an interesting result for the researchers who are using semiconducting SiCNTs for device applications.

\section{Conclusions}

In summary, the results show a reduction in threshold voltage in case of radial compression and axial elongation applied simultaneously in $(8,0)$ semiconducting SiCNT. High current conduction in deformed tube is associated with the introduction of new electronic states near the Fermi level, which is also attributed by the reduction in bandgap. At high bias voltages these electronic states are brought over the Fermi level which facilitates tunneling that results in increase in current in deformed semiconducting SiCNT structures.

\section{Acknowledgements}

The authors would like to thank Prof. RM Patrekar, ECE Department, VNIT, Nagpur, for his support in terms of providing access to the laboratory resources. The authors would also like to thank the editor and two anonymous referees for their comments and suggestions which have improved the clarity and the overall presentation of the results.

\section{References}

Alfieri G and Kimoto T 2010 Appl. Phys. Letts 97043108

Atomistix, QuantumWise A/S (www.quantumwise.com)

Brandbyge M, Mozos J L, Ordejón P, Taylor J and Stokbro K 2002 Phys. Rev. B65 165401

Choudhary S and Qureshi S 2011 J. Nano-Electron. Phys. 3584

Gali A 2006 Phys. Rev. B73 1

Lakshmi S, Dutta Sudipta and Pati Swapan K 2008 J. Phys. Chem. C112 14718

Larina E V, Chmyrev V I, Skorikov V M, D'yachkov P N and Makaev D V 2007 Inorg. Mater. 44823

Li Z and Kosov D S 2006 J. Phys. Chem. B110 9893 
Lu J Q, Wu J, Duan W, Liu F, Zhu B F and Gu B L 2003 Phys. Rev. Letts 90156601

Manna Arun K and Pati Swapan K 2010 Nanoscale 21190

Mavrandonakis A, Froudakis George E, Schnell M and Muhlhauser M M 2003 Nano Letts. 31481

Moradian R, Behzad S and Chegel R 2008 J. Phys. Condens. Matter 20465214

Mpourmpakis G, Froudakis George E, Lithoxoos George P and Samios J 2006 Nano Letts. 61581

Muralidharan Bhaskaran, Ghosh Avik W, Pati Swapan K and Datta Supriyo 2007 IEEE Trans. Nanotechnol. 6536

Nanotube Modeller, www.jcrystal.com

Raidongia K, Jagadeesan D, Upadhyay-Kahaly M, Waghmare U V, Pati Swapan K, Eswaramoorthya M and Rao C N R 2008 J. Mater. Chem. 1883

Ren Y, Chen K Q, Wan Q, Zou B S and Zhang Y 2009 Appl. Phys. Letts 94183506

Soler J M, Artacho E, Gale J D, García A, Junquera J, Ordejón P and Sánchez-Portal D 2002 J. Phys. Condens. Matter 142745
Song J, Yang Y and Liu H 2009 IEEE EDSSC 509

Taguchia T, Igawaa N and Yamamotoa H 2005a J. Am. Ceram. Soc. 88459

Taguchia T, Igawaa N, Yamamotoa H, Shamotoa S and Jitsukawa S 2005b Physica E 28431

Taylor J, Guo H and Wang J 2001 Phys. Rev. B63 245407

Voggu Rakesh, Pal Shrinwantu, Pati Swapan K and Rao C N R 2008 J. Phys. Condens. Matter 20215211

Wang Z, Zu X, Xiao H, Gao F and Weber W J 2008 Appl. Phys. Letts 92183116

Wu I J and Guo G Y 2007 Phys. Rev. B76 035343

Wu R Q, Yang M, Lu Y H and Feng Y P 2008 J. Phys. Chem. C112 15985

Xie Z, Tao D and Wang J 2007 Nanosci. Nanotechnol. 7647

Yamacli S and Avci M 2009 Phys. Lett. A374 297

Yang Y T, Ding R X and Song J X 2010 Physica B406 216

Zhao M, Xia Y, Li F, Zhang R Q and Lee S T 2005 Phys. Rev. B71 085312

Zheng Fawei, Yang Yu and Zhang Ping 2010 Appl. Phys. Letts 97 263105 